K.P.R. Rao, K. Bhanu Lakshmi and V.C.C. Raju / TJ MCS Vol .4 No.4 (2012) 585-590

The Journal of

Mathematics and Computer Science

Available online at

\title{
http://www.TIMCS.com
}

The Journal of Mathematics and Computer Science / TJMCS Vol .4 No.4 (2012) 585-590

\section{A Unique Common Fixed Point Theorem For Three Mappings in G -Cone metric spaces}

\author{
K.P.R. Rao \\ Department of Mathematics \\ Acharya Nagarjuna University \\ Nagarjuna Nagar-522 510, A.P., India \\ email: kprrao2004@yahoo.com \\ K. Bhanu Lakshmi \\ Science and Humanities Department \\ Lakireddy Balireddy College of Engineering \\ Mylavaram-521230,A.P., India \\ email: bhanulaks@gmail.com \\ V.C.C. Raju \\ Department of Mathematics, University of Botswana \\ Private Bag UB 00704, Gaborone, Botswana \\ email: varanasi@mopipi.ub.bw
}

Received: March 2012 , Revised: November 2012

Online Publication: November 2012

\section{Abstract}

In this paper we obtain a unique common fixed point theorem for three mappings in G-cone metric spaces and obtain an extension and improvement of a theorem of I. Beg et. al. [1].

Keywords: G - cone metric space, common fixed points, symmetric space.

2010 Mathematics Subject Classification: 47H10; 54H25 . 
K.P.R. Rao, K. Bhanu Lakshmi and V.C.C. Raju / TJ MCS Vol .4 No.4 (2012) 585-590

\section{Introduction and preliminaries}

Based on cone metric spaces introduced by [2] and on G-metric spaces introduced by [4], I.

Beg et. al. [1] introduced generalized cone metric spaces as follows:

Let $\mathrm{E}$ be a real Banach space and $\mathrm{P}$ be a subset of $\mathrm{E}$. The subset $\mathrm{P}$ is called a Cone if it has the following properties:

(i) $\mathrm{P}$ is non empty, closed and $\mathrm{P} \neq\{0\}$;

(ii) $0 \leq a, b \in R$ and $x, y \in P \Rightarrow a x+b y \in P$;

(iii) $\mathrm{P} \cap(-\mathrm{P})=\{0\}$.

For a given cone $\mathrm{P} \subseteq \mathrm{E}$, we can define a partial ordering $\leq$ on $\mathrm{E}$ with respect to $\mathrm{P}$ by $\mathrm{x} \leq \mathrm{y}$ if and only if $\mathrm{y}-\mathrm{x} \in \mathrm{P}$. We will write $\mathrm{x}<\mathrm{y}$ if $\mathrm{x} \leq \mathrm{y}$ and $\mathrm{x} \neq \mathrm{y}$, while $\mathrm{x} \ll \mathrm{y}$ will stands for $\mathrm{y}-\mathrm{x} \in \mathrm{P}^{0}$, where $\mathrm{P}^{0}$ denotes the interior of $\mathrm{P}$.

Proposition 1.1 ([5]). Let $\mathrm{P}$ be a cone in a real Banach space $E$. If $\mathrm{a} \in \mathrm{P}$ and $\mathrm{a} \leq \lambda \mathrm{a}$ for some $\lambda \in[0,1)$ then $\mathrm{a}=0$.

Proposition 1.2 ([3],Cor.1.4).Let $\mathrm{P}$ be a cone in a real Banach space E .

(i) If $\mathrm{a} \leq \mathrm{b}$ and $\mathrm{b}<<\mathrm{c}$, then $\mathrm{a}<<\mathrm{c}$

(ii) If $\mathrm{a} \in \mathrm{E}$ and $\mathrm{a}<<\mathrm{c}$ for all $\mathrm{c} \in \mathrm{P}^{0}$, then $\mathrm{a}=0$.

Remark 1.3 ([3]). $\lambda \mathrm{P}^{0} \subseteq \mathrm{P}^{0}$ for $\lambda>0$ and $\mathrm{P}^{0}+\mathrm{P}^{0} \subseteq \mathrm{P}^{0}$.

Definition 1.4 ([1]). Let $X$ be a nonempty set and let $G: X \times X \times X \rightarrow E$ be a function satisfying the

following properties :

$\left(G_{1}\right): G(x, y, z)=0$ if $x=y=z$,

$\left(G_{2}\right): 0<G(x, x, y)$ for all $x, y \in X$ with $x \neq y$,

$\left(G_{3}\right): G(x, x, y) \leq G(x, y, z)$ for all $x, y, z \in X$ with $y \neq z$,

$\left(G_{4}\right): G(x, y, z)=G(x, z, y)=G(y, z, x)=\ldots($ symmetry in three variables),

$\left(G_{5}\right): G(x, y, z) \leq G(x, a, a)+G(a, y, z)$ for all $x, y, z, a \in X$.

Then the function $G$ is called a generalized cone metric on $X$ and $X$ is called a generalized cone metric space or a $G$ - cone metric space. It is clear that if $G(x, y, z)=0$ then $x=y=z$ for any $\mathrm{x}, \mathrm{y}, \mathrm{z} \in \mathrm{X}$.

Definition 1.5 ([1]). A G - cone metric space $X$ is called symmetric if $G(x, x, y)=G(x, y, y)$ for all $x, y$ $\in \mathrm{X}$.

Definition $1.6([1])$. Let $X$ be a $G$ - cone metric space and $\left\{\mathrm{X}_{\mathrm{n}}\right\}$ be a sequence in $\mathrm{X}$. The sequence $\left\{\mathrm{X}_{\mathrm{n}}\right\}$ is said to converge to a point $\mathrm{x} \in \mathrm{X}$ if for every $\mathrm{c} \in \mathrm{E}$ with $0 \ll \mathrm{c}$ there is $\mathrm{N}$ such that $\mathrm{G}\left(\mathrm{x}_{\mathrm{n}}, \mathrm{x}_{\mathrm{m}}, \mathrm{x}\right)<<$ for all $n, m>N$. In this case, we write $x_{n} \rightarrow x$ as $n \rightarrow \infty$.

The sequence $\left\{x_{n}\right\}$ is said to be a $G$ - Cauchy sequence in $X$ if for every $c \in E$ with $0<<$ there is $\mathrm{N}$ such that $\mathrm{G}\left(\mathrm{x}_{\mathrm{n}}, \mathrm{x}_{\mathrm{m}}, \mathrm{x}_{\mathrm{l}}\right) \ll<$ for all $\mathrm{n}, \mathrm{m}, \mathrm{l}>\mathrm{N}$.

$\mathrm{X}$ is said to be complete if every $\mathrm{G}$ - Cauchy sequence in $\mathrm{X}$ is convergent in $\mathrm{X}$. 
Proposition 1.7 ([1],Lemma 2.8). Let $X$ be a $G$ - cone metric space. Then for a sequence $\left\{X_{n}\right\} \subseteq X$ and a point $\mathrm{x} \in \mathrm{X}$, the following are equivalent

(i) $\left\{\mathrm{x}_{\mathrm{n}}\right\}$ is $\mathrm{G}$ - convergent to $\mathrm{x}$,

(ii) $\mathrm{G}\left(\mathrm{x}_{\mathrm{n}}, \mathrm{x}_{\mathrm{n}}, \mathrm{x}\right) \rightarrow 0$ as $\mathrm{n} \rightarrow \infty$,

(iii) $\mathrm{G}\left(\mathrm{x}_{\mathrm{n}}, \mathrm{x}, \mathrm{x}\right) \rightarrow 0$ as $\mathrm{n} \rightarrow \infty$,

(iv) $\mathrm{G}\left(\mathrm{x}_{\mathrm{m}}, \mathrm{x}_{\mathrm{n}}, \mathrm{x}\right) \rightarrow 0$ as $\mathrm{n}, \mathrm{m} \rightarrow \infty$.

Proposition 1.8 ([1],Lemma 2.9). Let X be a G - cone metric space. Then the function $\mathrm{G}(\mathrm{x}, \mathrm{y}, \mathrm{z})$ is jointly continuous in all three of its variables.

Remark 1.9 ([5]). If $c \in \mathrm{P}^{0}, 0 \leq \mathrm{a}_{\mathrm{n}}$ and $\mathrm{a}_{\mathrm{n}} \rightarrow 0$, then there exists $\mathrm{n}_{0} \in \mathrm{N}$ such that for all $\mathrm{n}>\mathrm{n}_{0}$ we have $\mathrm{a}_{\mathrm{n}}<<\mathrm{c}$.

Ismat Beg et.al [1] proved the following

Theorem 1.10 ([1],Theorem 3.1). Let $\mathrm{X}$ be a complete symmetric $\mathrm{G}$ - cone metric space and $\mathrm{T}: \mathrm{X}$ $\rightarrow \mathrm{X}$ be a mapping satisfying one of the following conditions

$$
\begin{gathered}
G(T x, T y, T z) \leq a G(x, y, z)+b G(x, T x, T x)+c G(y, T y, T y)+d G(z, T z, T z) \\
\text { and } \\
G(T x, T y, T z) \leq a G(x, y, z)+b G(x, x, T x)+c G(y, y, T y)+d G(z, z, T z)
\end{gathered}
$$

for all $\mathrm{x}, \mathrm{y}, \mathrm{z} \in \mathrm{X}$, where $0 \leq \mathrm{a}+\mathrm{b}+\mathrm{c}+\mathrm{d}<1$.

Then $T$ has a unique fixed point in $\mathrm{X}$.

Now, we give a Lemma in G - cone metric spaces which is similar in cone metric spaces given by Jain et.al [ 6 ].

Lemma 1.11 : Let $X$ be a $G$ - cone metric space, $P$ be a cone in a real Banach space $E$ and $k_{1}, k_{2}, k_{3}, k_{4}$ $\geq 0$ such that $\mathrm{k}_{1}+\mathrm{k}_{2}+\mathrm{k}_{3}+\mathrm{k}_{4}>0$ and $\mathrm{k}>0$. If $\mathrm{x}_{\mathrm{n}} \rightarrow \mathrm{x}, \mathrm{y}_{\mathrm{n}} \rightarrow \mathrm{y}, \mathrm{z}_{\mathrm{n}} \rightarrow \mathrm{z}$ and $\mathrm{p}_{\mathrm{n}} \rightarrow \mathrm{p}$ in $\mathrm{X}$ and (1.11.1) ka $\leq k_{1} G\left(x_{n}, x_{m}, x\right)+k_{2} G\left(y_{n}, y_{m}, y\right)+k_{3} G\left(z_{n}, z_{m}, z\right)+k_{4} G\left(p_{n}, p_{m}, p\right)$ then $\mathrm{a}=0$.

Proof. Since $x_{n} \rightarrow x, y_{n} \rightarrow y, z_{n} \rightarrow z$ and $p_{n} \rightarrow p$, we have for $c \in P^{0}$, there exists a positive integer $N_{c}$ such that

$$
\begin{aligned}
& \frac{c}{k_{1}+k_{2}+k_{3}+k_{4}}-\mathrm{G}\left(\mathrm{x}_{\mathrm{n}}, \mathrm{x}_{\mathrm{m}}, \mathrm{x}\right), \frac{c}{k_{1}+k_{2}+k_{3}+k_{4}}-\mathrm{G}\left(\mathrm{y}_{\mathrm{n}}, \mathrm{y}_{\mathrm{m}}, \mathrm{y}\right), \\
& \frac{\mathrm{c}}{\mathrm{k}_{1}+\mathrm{k}_{2}+\mathrm{k}_{3}+\mathrm{k}_{4}}-\mathrm{G}\left(\mathrm{z}_{\mathrm{n}}, \mathrm{z}_{\mathrm{m}}, \mathrm{z}\right), \frac{\mathrm{c}}{\mathrm{k}_{1}+\mathrm{k}_{2}+\mathrm{k}_{3}+\mathrm{k}_{4}}-\mathrm{G}\left(\mathrm{p}_{\mathrm{n}}, \mathrm{p}_{\mathrm{m}}, \mathrm{p}\right) \in \mathrm{P}^{0} \forall \mathrm{n}>\mathrm{N}_{\mathrm{c}} .
\end{aligned}
$$

From Remark 1.3, we have

$$
\begin{aligned}
& \frac{\mathrm{k}_{1} \mathrm{c}}{\mathrm{k}_{1}+\mathrm{k}_{2}+\mathrm{k}_{3}+\mathrm{k}_{4}}-\mathrm{k}_{1} \mathrm{G}\left(\mathrm{x}_{\mathrm{n}}, \mathrm{x}_{\mathrm{m}}, \mathrm{x}\right), \frac{\mathrm{k}_{2} \mathrm{c}}{\mathrm{k}_{1}+\mathrm{k}_{2}+\mathrm{k}_{3}+\mathrm{k}_{4}}-\mathrm{k}_{2} \mathrm{G}\left(\mathrm{y}_{\mathrm{n}}, \mathrm{y}_{\mathrm{m}}, \mathrm{y}\right), \\
& \frac{\mathrm{k}_{3} \mathrm{c}}{\mathrm{k}_{1}+\mathrm{k}_{2}+\mathrm{k}_{3}+\mathrm{k}_{4}}-\mathrm{k}_{3} \mathrm{G}\left(\mathrm{z}_{\mathrm{n}}, \mathrm{z}_{\mathrm{m}}, \mathrm{z}\right), \frac{\mathrm{k}_{4} \mathrm{c}}{\mathrm{k}_{1}+\mathrm{k}_{2}+\mathrm{k}_{3}+\mathrm{k}_{4}}-\mathrm{k}_{4} \mathrm{G}\left(\mathrm{p}_{\mathrm{n}}, \mathrm{p}_{\mathrm{m}}, \mathrm{p}\right) \in \mathrm{P}^{0} \forall \mathrm{n}>\mathrm{N}_{\mathrm{c}} .
\end{aligned}
$$

Adding these four and by Remark 1.3, we have 
$c-\left[k_{1} G\left(x_{n}, x_{m}, x\right)+k_{2} G\left(y_{n}, y_{m}, y\right)+k_{3} G\left(z_{n}, z_{m}, z\right)+k_{4} G\left(p_{n}, p_{m}, p\right)\right] \in P^{0} \forall n>N_{c}$.

Now from(1.11.1) and Proposition 1.2(i), we have ka $<<\mathrm{c}$ for all $\mathrm{c} \in \mathrm{P}^{0}$.

By Proposition 1.2(ii), we have $\mathrm{a}=0$ as $\mathrm{k}>0$.

\section{Main result}

Theorem 2.1 . Let $(X, G)$ be a symmetric $G$-cone metric space and $A, B, C: X \rightarrow X$ be satisfying

$$
G(A x, B y, C z) \leq k \max \left\{\begin{array}{c}
G(x, y, z), G(x, A x, B y), \\
G(y, B y, C z), G(z, C z, A x), \\
G(x, A x, A x), G(y, B y, B y), G(z, C z, C z),
\end{array}\right\}
$$

for all $\mathrm{x}, \mathrm{y}, \mathrm{z} \in \mathrm{X}$, where $0 \leq \mathrm{k}<1$.

Then the mappings $\mathrm{A}, \mathrm{B}$ and $\mathrm{C}$ have a unique common fixed point in $\mathrm{X}$.

Proof. Choose $\mathrm{x}_{0} \in X$. Define $\mathrm{x}_{3 n+1}=A x_{3 n}, \mathrm{x}_{3 n+2}=B x_{3 n+1}, x_{3 n+3}=C x_{3 n+2}, n=0,1,2, \ldots \ldots$

Case(I) If $x_{3 n}=x_{3 n+1}$ then $x_{3 n}$ is a fixed point of A. Denote $x_{3 n}=x$. Then $A x=x$.

Suppose $B x \neq C x$. Then from (2.1.1)

$$
\begin{aligned}
G(x, B x, C x) & =G(x, B x, C x) \\
& \leq k \max \left\{\begin{array}{c}
0, G(x, x, B x), G(x, B x, C x), G(x, C x, x), \\
0, G(x, B x, B x), G(x, C x, C x)
\end{array}\right\} \\
& =k \max \{G(x, x, B x), G(x, B x, C x), G(x, x, C x)\} \ldots(1) \text {, as } X \text { is symmetric } \\
& \leq k G(x, B x, C x) \quad \text { from }\left(G_{3}\right)
\end{aligned}
$$

It is a contradiction. Hence $\mathrm{Bx}=\mathrm{Cx}$.

Now from(1),G(x,Bx,Bx) $\leq \mathrm{k} G(\mathrm{x}, \mathrm{Bx}, \mathrm{Bx})$.

Now from Proposition 1.1, $\mathrm{Bx}=\mathrm{x}$. Hence $\mathrm{Cx}=\mathrm{x}$.

Thus $\mathrm{x}$ is a common fixed point of $\mathrm{A}, \mathrm{B}$ and $\mathrm{C}$.

Suppose $\mathrm{x}^{1}$ is another common fixed point of $\mathrm{A}, \mathrm{B}$ and $\mathrm{C}$. Then

$$
\begin{aligned}
G\left(x, x, x^{1}\right) & =G\left(A x, B x, C x^{1}\right) \\
& \leq k \max \left\{G\left(x, x, x^{1}\right), 0, G\left(x, x, x^{1}\right), G\left(x^{1}, x^{1}, x\right), 0,0,0\right\} \\
& =k G\left(x, x, x^{1}\right) \text { as } X \text { is symmetric }
\end{aligned}
$$

Hence $\mathrm{x}=\mathrm{x}^{1}$. Thus $\mathrm{x}$ is the unique common fixed point of $\mathrm{A}, \mathrm{B}$ and $\mathrm{C}$.

Similarly, if $x_{3 n+1}=x_{3 n+2}$ or $x_{3 n+2}=x_{3 n+3}$ then we can show that $A, B$ and

$\mathrm{C}$ have a unique common fixed point in $\mathrm{X}$.

Case(II): Assume that $\mathrm{x}_{\mathrm{n}} \neq \mathrm{x}_{\mathrm{n}+1}$ for all $\mathrm{n}$.

As $\mathrm{X}$ is symmetric and from $\left(\mathrm{G}_{3}\right)$,we have

$$
\begin{aligned}
G\left(x_{3 n+1}, x_{3 n+2}, x_{3 n+3}\right) & \\
= & G\left(A x_{3 n}, B x_{3 n+1}, C x_{3 n+2}\right) \\
\leq & k \max \left\{\begin{array}{c}
G\left(x_{3 n}, x_{3 n+1}, x_{3 n+2}\right), G\left(x_{3 n}, x_{3 n+1}, x_{3 n+2}\right), G\left(x_{3 n+1}, x_{3 n+2}, x_{3 n+3}\right) \\
G\left(x_{3 n+2}, x_{3 n+3}, x_{3 n+1}\right), G\left(x_{3 n}, x_{3 n+1}, x_{3 n+1}\right) \\
G\left(x_{3 n+1}, x_{3 n+2}, x_{3 n+2}\right), G\left(x_{3 n+2}, x_{3 n+3}, x_{3 n+3}\right)
\end{array}\right\}
\end{aligned}
$$


K.P.R. Rao, K. Bhanu Lakshmi and V.C.C. Raju / TJ MCS Vol .4 No.4 (2012) 585-590

$$
\begin{aligned}
& \leq \quad k \max \left\{\begin{array}{c}
G\left(x_{3 n}, x_{3 n+1}, x_{3 n+2}\right), G\left(x_{3 n+1}, x_{3 n+2}, x_{3 n+3}\right) \\
G\left(x_{3 n}, x_{3 n}, x_{3 n+1}\right), G\left(x_{3 n+1}, x_{3 n+2}, x_{3 n+2}\right), G\left(x_{3 n+2}, x_{3 n+3}, x_{3 n+3}\right)
\end{array}\right\} \\
& \leq \quad k \max \left\{\begin{array}{c}
G\left(x_{3 n}, x_{3 n+1}, x_{3 n+2}\right), G\left(x_{3 n+1}, x_{3 n+2}, x_{3 n+3}\right) \\
G\left(x_{3 n}, x_{3 n+1}, x_{3 n+2}\right), G\left(x_{3 n+1}, x_{3 n+2}, x_{3 n}\right), G\left(x_{3 n+2}, x_{3 n+3}, x_{3 n+1}\right)
\end{array}\right\}
\end{aligned}
$$

Thus $G\left(x_{3 n+1}, x_{3 n+2}, x_{3 n+3}\right) \leq k$ G( $\left.x_{3 n}, x_{3 n+1}, x_{3 n+2}\right)$.

Similarly, we can show that $G\left(x_{3 n+2}, x_{3 n+3}, x_{3 n+4}\right) \leq k G\left(x_{3 n+1}, x_{3 n+2}, x_{3 n+3}\right)$

and $G\left(x_{3 n+3}, x_{3 n+4}, x_{3 n+5}\right) \leq G\left(x_{3 n+2}, x_{3 n+3}, x_{3 n+4}\right)$.

Thus $G\left(x_{n}, x_{n+1}, x_{n+2}\right) \leq k G\left(x_{n-1}, x_{n}, x_{n+1}\right), n=1,2,3, \ldots$.

Hence

$\mathrm{G}\left(\mathrm{x}_{\mathrm{n}}, \mathrm{x}_{\mathrm{n}+1}, \mathrm{x}_{\mathrm{n}+2}\right) \leq \quad \mathrm{kG}\left(\mathrm{x}_{\mathrm{n}-1}, \mathrm{x}_{\mathrm{n}}, \mathrm{x}_{\mathrm{n}+1}\right)$

$\leq \quad k^{2}\left(G\left(x_{n-2}, x_{n-1}, x_{n}\right)\right.$

From $\left(\mathrm{G}_{3}\right)$ and (2), we have

$\mathrm{k}^{\mathrm{n}}\left(\mathrm{G}\left(\mathrm{x}_{0}, \mathrm{x}_{1}, \mathrm{x}_{2}\right)\right)$

$\mathrm{G}\left(\mathrm{x}_{\mathrm{n}}, \mathrm{x}_{\mathrm{n}}, \mathrm{x}_{\mathrm{n}+1}\right) \leq \mathrm{G}\left(\mathrm{x}_{\mathrm{n}}, \mathrm{x}_{\mathrm{n}+1}, \mathrm{x}_{\mathrm{n}+2}\right) \leq \mathrm{k}^{\mathrm{n}}\left(\mathrm{G}\left(\mathrm{x}_{0}, \mathrm{x}_{1}, \mathrm{x}_{2}\right)\right)$.

Now for $m>n$,

$\mathrm{G}\left(\mathrm{x}_{\mathrm{n}}, \mathrm{x}_{\mathrm{n}}, \mathrm{x}_{\mathrm{m}}\right)$

$$
\begin{array}{ll}
\leq & G\left(x_{n}, x_{n}, x_{n+1}\right)+G\left(x_{n+1}, x_{n+1}, x_{n+2}\right)+\ldots+G\left(x_{m-1}, x_{m-1}, x_{m}\right) \\
\leq & k^{n} G\left(x_{0}, x_{1}, x_{2}\right)+k^{n+1} G\left(x_{0}, x_{1}, x_{2}\right)+\ldots+k^{m-1} G\left(x_{0}, x_{1}, x_{2}\right) \\
\leq & \quad \frac{k^{n}}{1-k} G\left(x_{0}, x_{1}, x_{2}\right) \\
& \rightarrow \quad 0 \text { as } n \rightarrow \infty .
\end{array}
$$

From Remark 1.9, it follows that for $0<<$ c and large $\mathrm{n}, \frac{\mathrm{k}^{\mathrm{n}}}{1-\mathrm{k}} \mathrm{G}\left(\mathrm{x}_{0}, \mathrm{x}_{1}, \mathrm{x}_{2}\right) \ll c$.

Now from Corollary 1.2(i), we have $\mathrm{G}\left(\mathrm{x}_{\mathrm{n}}, \mathrm{x}_{\mathrm{n}}, \mathrm{x}_{\mathrm{m}}\right)<\mathrm{c}$ for all $\mathrm{m}>\mathrm{n}$. Hence $\left\{\mathrm{x}_{\mathrm{n}}\right\}$ is $\mathrm{G}-$ Cauchy.

Since $X$ is $G$ - complete, there exists $p \in X$ such that $x_{n} \rightarrow p$ as $n \rightarrow \infty$.

Now

G(Ap, p, p)

$$
\begin{array}{ll}
\leq & G\left(A p, B x_{3 n+1}, B x_{3 n+1}\right)+G\left(B x_{3 n+1}, p, p\right) \\
\leq & G\left(B x_{3 n+1}, C x_{3 n+2}, C x_{3 n+2}\right)+G\left(C x_{3 n+2}, A p, B x_{3 n+1}\right)+G\left(B x_{3 n+1}, p, p\right) \\
\leq & G\left(x_{3 n+2}, x_{3 n+3}, x_{3 n+3}\right)+G\left(x_{3 n+2}, p, p\right)+G\left(A p, B x_{3 n+1}, C x_{3 n+2}\right) \\
\leq & \quad k \max \left\{\begin{array}{c}
G\left(p, x_{3 n+1}, x_{3 n+2}\right), G\left(p, A p, x_{3 n+2}\right), \\
G\left(x_{3 n+1}, x_{3 n+2}, x_{3 n+3}\right), G\left(x_{3 n+2}, x_{3 n+3}, A p\right), \\
G(p, A p, A p), G\left(x_{3 n+1}, x_{3 n+2}, x_{3 n+2}\right), G\left(x_{3 n+2}, x_{3 n+3}, x_{3 n+3}\right)
\end{array}\right\} \\
& k \max \left\{\begin{array}{c}
G\left(p, x_{3 n+2}, p, p\right)+G\left(p, x_{3 n+3}, x_{3 n+3}\right)+ \\
G\left(x_{3 n+2}, p, p\right)+G\left(p, x_{3 n+1}, x_{3 n+3}\right), G(A p, p, p)+G\left(p, x_{3 n+2}, x_{3 n+3}\right), \\
G(p, p, A p), G\left(x_{3 n+2}, p, p\right)+G\left(p, x_{3 n+1}, x_{3 n+2}\right), \\
G\left(x_{3 n+2}, p, p\right)+G\left(p, x_{3 n+3}, x_{3 n+3}\right)
\end{array}\right\}
\end{array}
$$

Thus we have

$G(A p, p, p) \leq 2 G\left(x_{3 n+2}, p, p\right)+G\left(p, x_{3 n+3}, x_{3 n+3}\right)+k G\left(p, x_{3 n+1}, x_{3 n+2}\right)$ or $(1-k) G(A p, p, p) \leq(2+k) G\left(x_{3 n+2}, p, p\right)+G\left(p, x_{3 n+3}, x_{3 n+3}\right)$ or

$G(A p, p, p) \leq(2+k) G\left(x_{3 n+2}, p, p\right)+G\left(p, x_{3 n+3}, x_{3 n+3}\right)+k G\left(p, x_{3 n+1}, x_{3 n+3}\right)$ or $(1-k) G(A p, p, p) \leq 2 G\left(x_{3 n+2}, p, p\right)+G\left(p, x_{3 n+3}, x_{3 n+3}\right)+k G\left(p, x_{3 n+2}, x_{3 n+3}\right)$ or $(1-k) G(A p, p, p) \leq 2 G\left(x_{3 n+2}, p, p\right)+G\left(p, x_{3 n+3}, x_{3 n+3}\right)$ or 
$G(A p, p, p) \leq(2+k) G\left(x_{3 n+2}, p, p\right)+G\left(p, x_{3 n+3}, x_{3 n+3}\right)+k G\left(p, x_{3 n+1}, x_{3 n+2}\right)$ or

$G(A p, p, p) \leq(2+k) G\left(x_{3 n+2}, p, p\right)+(1+k) G\left(p, x_{3 n+3}, x_{3 n+3}\right)$.

Now from Proposition 1.7 and from Lemma 1.11, it follows thatG(Ap, $p, p)=0$ so that $A p=p$.

The rest of the proof follows as in Case(I).

\section{References.}

[1] Ismat Beg, Mujahid Abbas and Talat Nazir, Generalized cone metric spaces, J. Nonlinear Sci. Appl. 3, No.1 (2010), 21 - 31.

[2] L.G. Huang and X. Zhang, Cone metric spaces and fixed point theorems of contractive mappings, J.Math.Anal.Appl., 332(2) (2007), 1468 - 1476.

[3] S. Rezapour and R. Hamlbarani, Some notes on the paper "Cone metric spaces and fixed point theorems of contractive mappings ",J.Math.Anal. Appl., 345 (2008), 719-724.

[4] Z. Mustafa and B. Sims, A new approach to generalized metric spaces, Journal of Nonlinear and Convex Analysis,Vol.7, no.2, (2006), 289 - 297.

[5] G. Jungck, S. Radenovic ,S. Radojevic and V. Rakocevic, Common fixed point theorems for weakly compatible pairs on cone metric spaces, Fixed point theory and Applications,Vol.2009,Article ID 643840,13 pages, doi:10.1155/2009/643840.

[6] Shoba Jain, Shishir Jain and Lal Bahadur, Compatibility and weak compatibility for four self maps in a cone metric space, Bulletin of Mathematical Analysis and Applications,Vol.2,Issue 1 (2010), $15-24$. 